\title{
Escala de actitudes hacia la investigación (EACIN): Evaluación de sus propiedades psicométricas en una muestra colombiana*
}

\author{
Attitudes Towards Research Scale (ATRS): Evaluation of its \\ Psychometric Properties in a Colombian Sample
}

Gloria Marlen Aldana de Becerra 1 ORCID - $\underline{\text { CvLAC }}$, Doris Amparo Babativa Novoa 2 ORCID - $\underline{\text { CvLAC }}$, Gilma Jeannette Caraballo Martínez ${ }^{3} \underline{\text { ORCID }}-\underline{\text { CvLAC }}$, César Armando Rey Anacona 4 ORCID - CVLAC

\author{
${ }^{123}$ Fundación Universitaria del Área Andina \\ ${ }^{4}$ Universidad Pedagógica y Tecnológica de Colombia \\ Colombia
}

Fecha correspondencia: Recibido: noviembre 22 de 2018. Aceptado: octubre 23 de 2019.

Forma de citar:

Aldana, G.M., Babativa, D.A, Caraballo, G.J, \& Rey, C.A. (2020). Escala de actitudes hacia la investigación (EACIN): evaluación de sus propiedades psicométricas en una muestra colombiana. Rev. CES Psico, 13(1), 89-103.

\section{Open access \\ (c) Copyright \\ Licencia creative commons \\ Etica de publicaciones \\ Revisión por pares \\ Gestión por Open Journal System DOl: http://dx.doi.org/10.21615/ cesp.13.1.6 \\ ISSN: 2011-3080}

Sobre el artículo:

* Este estudio es un producto del proyecto de investigación "Actitudes hacia la investigación de estudiantes de salud y ciencias económicas de

Comparte

\section{Resumen}

El objetivo del presente estudio fue examinar las propiedades psicométricas de la Escala de Actitudes hacia la Investigación (EACIN) en una muestra de estudiantes, docentes y directivos colombianos, el principal resultado fue una versión revisada de la misma (EACIN-R). Participaron 427 personas (261 mujeres y 166 hombres), entre 16 y 56 años de edad ( $X=25,79$ años, $D X=8,46$ años). El instrumento había sido validado previamente en su contenido por ocho jueces y obtenido un alfa de Cronbrach de 0,85. Para la evaluación de las propiedades psicométricas se utilizó el análisis paralelo de Horn que permitió identificar tres factores que incluyen 28 ítems agrupados en las siguientes sub-escalas conforme al contenido de sus ítems: (a) desinterés por la investigación ( $a=0,87)$; (b) vocación por la investigación $(a=0,87)$ y (c) valoración de la investigación $(a=0,77)$. El alfa de Cronbrach total fue de 0,87 , que evidencia un buen nivel de consistencia interna. Se recomienda continuar estudiando las propiedades psicométricas del instrumento, particularmente su estabilidad y su validez de criterio, para posteriormente obtener baremos teniendo en cuenta variables como el sexo, la edad y el nivel educativo.

Palabras clave: Actitudes, Investigación, Fiabilidad, Validez, Estudiantes Universitarios.

\section{Abstract}

The purpose of this study was to examine the psychometric properties of the Escala de Actitudes Hacia la Investigación (Attitudes Towards Research Scale) in a sample of Colombian students, teachers and managers, which resulted in a revised version of the scale (EACIN-R). The sample consisted of 427 persons (261 women and 166 men), aged between 15 and 65 ( $X=25.79$ years, $D X=8.46$ years). The instrument was validated previously in its content by eight judges $(\boldsymbol{a}=.85)$. Horn's parallel analysis was used to identify three factors which included 28 items grouped in the following subscales according to the content of their items: (a) disinterest in research ( $a=.87)$; (b) vocation for research $(\alpha=.87)$, and (c) research valuation $(\alpha=.77)$. Total alpha 
dos instituciones educativas colombianas", código CV2015050, aprobado y financiado por la Fundación Universitaria del Área Andina (FUAA), en colaboración con el Grupo de Investigación en Psicología Clínica y de la Salud de la Universidad Pedagógica y Tecnológica de Colombia (UPTC).

\section{Sobre los autores:}

1. Estudiante de Doctorado en Educación. Magister en Educación y Desarrollo Social. Especialista en Teorías, Métodos y Técnicas de Investigación Social. Especialista en Docencia Universitaria. Psicóloga. Líder del Grupo de Investigación Procesos Psicosociales, de la Fundación Universitaria del Área Andina - sede Bogotá.

2. Estudiante de Doctorado en Psicología. Magister en Docencia Universitaria. Psicóloga. Integrante del Grupo de Investigación Estrategias Administrativas, de la Fundación Universitaria del Área Andina - sede Bogotá.

3. Magister en Investigación Social Interdisciplinaria. Fonoaudióloga. Integrante del Grupo de Investigación Procesos Psicosociales, de la Fundación Universitaria del Área Andina -sede Bogotá.

4. Doctor en Psicología Clínica y de la Salud. Psicólogo. Líder del Grupo de Investigación en Psicología Clínica y de la Salud, Universidad Pedagógica y Tecnológica de Colombia. was .87, which shows a good level of internal consistency. It is suggested further study about the psychometric properties of the instrument, particularly its stability and criterion validity, in order to obtain subsequently normalized scores having account variables as sex, age and education level.

Key words: Attitudes, Research, Reliability, Validity, University Students.

\section{Introducción}

La investigación constituye una estrategia de enseñanza/aprendizaje en la formación de profesionales, en cuanto contribuye a potenciar la capacidad metacognitiva para aprender a aprender, disposición a problematizar hechos de la realidad y del propio desempeño como estudiante o profesional. No solo se constituye en una actividad fundamental para la obtención de nuevos conocimientos, sino que también se convierte en una herramienta para la formación de profesionales con alto nivel académico.

Sin embargo, diversas situaciones como el excesivo formalismo de los procesos de investigación, la docencia enfocada más a la transmisión de conocimiento que a la investigación, el aprendizaje memorístico, los escasos recursos para investigar, la falta de tiempo y experticia de los docentes para apoyar a los estudiantes para que investiguen, así como las dificultades en las tareas de lectoescritura y baja producción de los estudiantes son algunos factores que influyen negativamente en las actividades de investigación en las instituciones de educación superior (IES) (Hacieminoglu, 2016; May \& Hong, 2015; Osman, 2016; Van Aalderen-Smeets, Walma van der Molen, van Hest, \& Poortman, 2017; Verkade \& Hoon, 2016). Igualmente, las condiciones, habilidades y actitudes de los diferentes actores involucrados juegan un papel importante cuando se trata de asumir la investigación como parte de la formación académica y del desempeño profesional.

Para Nobigrot-Kleinman, Nobigrot-Streimbleinsky y Galván-Huerta (1995) promover actitudes positivas hacia la investigación y hacia el aprendizaje hace parte de los objetivos curriculares; y desarrollar estas predisposiciones contribuirá a que el estudiante logre, entre otros aspectos, mayor comprensión de la naturaleza del proceso científico, propiciando en él un abordaje de los problemas en forma crítica y organizada, y transfiriendo esas actitudes a situaciones de la vida cotidiana.

Según Mamani (2011) el estudio de las actitudes hacia la investigación permite identificar contextos propicios para la construcción de conocimiento, formación de investigadores y generación de condiciones positivas para la investigación, orientada a la producción de conocimientos y a la solución de problemas. Igualmente, da lugar a identificar la predisposición de los individuos hacia la actividad científica (De la Cruz, 2013), encaminar a quienes la tengan a dedicar su trayectoria laboral en este sentido, y detectar las actitudes y capacidades que deben fortalecerse para formar investigadores que realicen y promuevan la investigación científica.

Las actitudes se definen como una disposición psicológica, adquirida, organizada y persistente, conformada por los componentes afectivo, cognitivo y conductual, que predisponen a los individuos a reaccionar preferentemente de una manera determinada, generando sentimientos y pensamientos agradables o desagradables hacia el objeto de la actitud, aprobándolo o desaprobándolo (Alonso, Alonso, \& Valadez. 2015; De las Salas, Perozo, \& Lago, 2014; Rodríquez \& Mora, 2016). Para Veliz (2017), apoyado en las tesis de Allportt (1935), la actitud es un estado mental y neuronal de 
Pág 91

Las actitudes, aunque pueden cambiar con el tiempo, tienen un fuerte componente predictivo, de manera que, si se conocen las actitudes de una persona hacia un objeto o situación, es posible inferir cuál será su conducta (Kennedy, Quinn, \& Taylor, 2016); por consiguiente, la posibilidad de predicción de las actitudes sobre las conductas reales radica en la evaluación de las mismas (Arellano Torres, Gaeta González, Peralta López, \& Cavazos Arroyo, 2019; Ortega Carrasco, Veloso Toledo, \& Hansen, 2018; Quezada-Berumen, Moral de la Rubia, \& Landero-Hernández, 2019). disposición para responder, organizado por la experiencia, que ejerce una influencia directiva o dinámica, sobre la conducta respecto a todos los objetos y situaciones con los que se relaciona. También es entendida como una disposición mental que incide en el aprendizaje, en la forma de representar y actuar en diferentes situaciones; y se le atribuye una función mediadora entre la persona y el ambiente (Rojas, Méndez, \& Rodríguez, 2012).

Las actitudes, aunque pueden cambiar con el tiempo, tienen un fuerte componente predictivo, de manera que, si se conocen las actitudes de una persona hacia un objeto o situación, es posible inferir cuál será su conducta (Kennedy, Quinn, \& Taylor, 2016); por consiguiente, la posibilidad de predicción de las actitudes sobre las conductas reales radica en la evaluación de las mismas (Arellano Torres, Gaeta González, Peralta López, \& Cavazos Arroyo, 2019; Ortega Carrasco, Veloso Toledo, \& Hansen, 2018; Quezada-Berumen, Moral de la Rubia, \& Landero-Hernández, 2019).

Por otra parte, Arias (2012) define la investigación como un proceso intencionado, metódico y riguroso, mediante el cual se obtiene conocimiento nuevo, objetivo, sistemático, organizado y verificable, orientado a la solución de problemas o a resolver interrogantes de carácter científico. Las actitudes hacia la investigación constituyen una organización duradera y persistente de predisposiciones con respecto a esta actividad, como la convicción de que el conocimiento no es un proceso acabado sino en continua construcción, que requiere disposición positiva para trabajar en equipo y en forma sistemática, modestia hacia el conocimiento que se posee y rectitud intelectual, entre otros (De las Salas, Perozo \& Lugo, 2014).

La importancia de medir las actitudes hacia la investigación de los docentes y estudiantes para la consolidación de la cultura investigativa en las IES ha motivado el desarrollo de diferentes instrumentos de evaluación en diversas poblaciones y desde diferentes enfoques.

Akçöltekin (2016) adaptó la Escala de Actitud de la Investigación Científica (Scientific Research Attitude Scale), de Korkmaz, Sahin y Yeșil (2011), que mide las actitudes de los profesores hacia la investigación científica. Para tal efecto, determinó su validez de contenido mediante el examen de cinco profesores de cuatro universidades, expertos en metodología de investigación científica, administró la Escala a 378 profesores de secundaria y obtuvo un coeficiente alfa de Cronbach de 0,86. La Escala de Actitudes hacia la Investigación (Attitudes Toward Research Scale), desarrollada en Chipre por Papanastasiou (2005), dirigida a estudiantes de pregrado, evalúa tres dominios: (a) valor del conocimiento científico (b) valor de la metodología científica y (c) valor de la ciencia. Esta Escala se aplicó a 226 estudiantes, en su mayoría mujeres (85\%), que realizaron un curso de metodología de la investigación obligatorio para la obtención del grado; el alfa de Cronbach fue de 0,95; el autor no menciona índices de validez. Posteriormente, Papanastasiou y Schumacker (2014) valoraron las propiedades psicométricas de este instrumento con una muestra de 541 estudiantes de la carrera de educación, en su mayoría mujeres (90,4\%), y obtuvieron un índice de fiabilidad de 0,94 con el modelo de Rasch, y tampoco reportaron índices de validez.

El instrumento diseñado y validado en Colombia por Castro (2017) para medir la actitud hacia la investigación formativa en estudiantes de pregrado, consta de 26 ítems agrupados en cinco factores: satisfacción-agrado por la investigación, apropiación conceptual, comportamientos de aprendizaje, exploración sistemática y habilidades percibidas. Fue validado a nivel de contenido por cinco revisores expertos, la fiabilidad 
obtenida con índice alfa de Cronbach fue de 0,83 en una muestra de 310 estudiantes de una universidad pública. Los cinco factores del instrumento explicaron el $56 \%$ de la varianza total.

El Índice de Actitud hacia la Investigación en Estudiantes de Pregrado, realizado en Colombia por Rojas et al. (2012), es un instrumento multipropósito que consta de 17 ítems. Evalúa tres dimensiones: (a) autoevaluación (b) incidencia de los profesores en las actitudes de los estudiantes e (c) incidencia de la institución en este tipo de actitudes. El índice alfa de Cronbach reportado fue de 0,88 en una muestra de 365 estudiantes de pregrado, los investigadores no reportaron índices de validez.

El Cuestionario para medir conocimientos, actitudes y prácticas de investigación de los estudiantes de pregrado de facultades de medicina de Perú, de Díaz, Manrique, Galán y Apolaya (2008), consta de tres componentes: (a) información general (b) conocimientos de los estudiantes del sistema de investigación en su facultad y (c) actitudes acerca de la investigación. Fue administrado a 1, 484 estudiantes de medicina y no se reportaron índices de validez ni de confiabilidad.

La Escala de actitud hacia el proceso de investigación científico social, realizada en Venezuela por Blanco y Alvarado (2005), evalúa la actitud hacia la investigación social, entendida como el conjunto de creencias, valores y conocimientos de los conceptos teóricos y metodológicos implicados en los diferentes momentos de la investigación social. Es una Escala tipo Likert compuesta por 20 ítems, producto del análisis de validez discriminante; distribuidos en tres dimensiones: afectiva, cognitiva y conductual. Las autoras aplicaron la Escala a 40 docentes e investigadores de Venezuela y reportaron un alfa de 0,86, pero no informaron índices de validez.

La Escala de Actitudes hacia la Investigación (EACIN), de Aldana, Caraballo, y Babativa (2016), contiene 34 ítems tipo Likert que examinan los componentes afectivo, cognitivo y conductual. La confiabilidad hallada con el índice alfa de Cronbach en una muestra de 187 participantes fue de 0,85 , que indica una buena consistencia interna. La principal ventaja de esta escala respecto a los instrumentos anteriormente mencionados es su cobertura, debido a que está diseñada para aplicar a todos los actores académicos: estudiantes desde educación primaria hasta postgrado, egresados y directivos académicos, puesto que los ítems evalúan aspectos generales de la investigación y son fáciles de comprender. Sin embargo, y siguiendo las recomendaciones de las autoras, es pertinente continuar el proceso de validación de las propiedades psicométricas de la escala y con muestras más grandes.

La versión EACIN-32, producto de la validación de la EACIN, realizada por Quezada-Berumen, Moral de la Rubia y Lanchero-Hernández (2019), en una muestra de 392 estudiantes de psicología de una universidad privada de México, arrojó tres factores: afectivo-conductual, con un alfa de Cronbach de 0,90; cognoscitivo, con un alfa de 0,89 y conductual-afectivo, con un alfa de 0,87. El alfa del total de la prueba fue de 0,92. Como la Escala cumplió con los criterios de consistencia interna, validez convergente y validez divergente, los autores recomiendan su aplicación en contextos universitarios. Y aunque la tasa de participantes fue del 10\% de la población de estudio, los autores manifiestan que las inferencias se limitan a estudiantes de la facultad de Psicología en la que se recolectó la muestra y recomiendan, entre otros aspectos, continuar el proceso de validación con el fin de aportar nuevas pruebas de validez del instrumento. 
Conforme a lo anterior, el objetivo del presente estudio fue evaluar las propiedades psicométricas de la versión original de la EACIN en una muestra de estudiantes, docentes y directivos académicos colombianos.

\section{Método}

\section{Diseño}

Se llevó a cabo un estudio instrumental cuyo fin es el desarrollo de pruebas, incluyendo su diseño o adaptación y la evaluación de sus propiedades psicométricas (Montero \& León, 2007). En este estudio, en particular, se examinaron las propiedades psicométricas de una escala para medir las actitudes hacia la investigación.

\section{Participantes}

La muestra estuvo constituida por 427 participantes seleccionados según su disponibilidad en tres instituciones educativas: (a) 52\% ( $n=220$ ) de la Universidad Pedagógica y Tecnológica de Colombia (UPTC), sede Tunja, (b) 40\% ( $n=173$ ) de la Fundación Universitaria del Área Andina (FUAA), sede Bogotá y (c) $8 \%$ ( $n=34)$ del Colegio Gimnasio Santa María del Alcázar (Bogotá), de los cuales 61\% ( $n=261)$ eran mujeres y 39\% $(n=166)$ hombres, con edades entre los 16 y los 56 años. El estado civil más reportado fue soltero $(n=349,82 \%)$, seguido de casado $(n=41,9 \%)$, unión libre $(n=27,6 \%)$, separado $(n=9$, $2 \%)$ y viudo $(n=1,0.2 \%)$.

El $8 \%(n=34)$ de participantes eran estudiantes de educación media vinculados al Colegio Gimnasio Santa María del Alcázar, mientras que 83,4\% ( $n=356)$ eran estudiantes universitarios adscritos a la FUAA y la UPTC. El 3\% $(n=13)$ eran directivos de la FUAA y 5,6\% ( $n=24)$ docentes de la misma institución.

En relación con los estudiantes universitarios, 17\% ( $n=72)$ eran alumnos de las siguientes carreras de la Facultad de Ciencias de la Salud de la FUAA: (a) Enfermería ( $n=18)$, (b) Instrumentación Quirúrgica $(n=29)$, (c) Terapia Respiratoria $(n=15)$ y (d) Optometría $(n=10)$, mientras que $14 \%(n=64)$ eran estudiantes de las siguientes carreras de la Facultad de Ciencias Administrativas, Económicas y Financieras de la misma institución: (e) Administración de Empresas ( $n=55)$, (f) Mercadeo y Publicidad $(n=4)$ y $(\mathrm{g})$ Negocios Internacionales $(n=5)$.

El 26\% ( $n=114)$ eran estudiantes de las siguientes carreras de la Facultad de Ciencias de la Salud de la UPTC: (a) Medicina $(n=46)$, (b) Enfermería ( $n=5)$ y (c) Psicología $(n=63)$, mientras que $27 \%(n=106)$ eran alumnos de las siguientes carreras de la Facultad de Ciencias Económicas y Administrativas de la misma universidad: (d) Administración de Empresas ( $n=57)$, (e) Contaduría ( $n=27)$ y (f) Economía $(n=22)$.

El tamaño muestral obedeció a la necesidad de contar con al menos diez participantes por cada ítem para la realización del análisis factorial del instrumento, que en total tiene 34 elementos, de conformidad con lo recomendado por Norman y Streiner (1996).

Los criterios de inclusión fueron: (a) estar matriculado formalmente en alguna de las instituciones, universidades y colegio, mencionadas; ser directivo o docente de alguna de las dos instituciones de educación superior participantes en el estudio, y (b) decidir participar voluntariamente en el estudio. 
Los criterios de exclusión fueron no cumplir los requisitos anteriores y, en el caso de los estudiantes de colegio, que sus padres o cuidadores no firmaran el consentimiento informado y/o que los estudiantes no firmaran el respectivo asentimiento.

\section{Instrumento}

Escala de Actitudes hacia la Investigación (EACIN), de Aldana et al. (2016), construida a partir de la escala para medir actitudes hacia la investigación realizada por Aldana y Joya (2011). Es una escala tipo Likert basada en el modelo multidimensional y está compuesta por 34 ítems distribuidos en tres componentes: (a) afectivo, entendido como lo que el sujeto siente o las emociones que le produce la investigación; (b) cognitivo, lo que el sujeto sabe o cree saber acerca de la investigación y (c) conductual, aquello que el sujeto hace o está dispuesto a hacer con respecto a la investigación. La validación de contenido se obtuvo mediante ocho jueces expertos, cuatro con grado de doctorado y cuatro de maestría, todos con experiencia en investigación y publicaciones producto de investigación, según lo reportado en sus hojas de vida registradas a través del CVLAC1.

La muestra estuvo conformada por 187 participantes de cuatro instituciones universitarias de Bogotá, 88 estudiantes de pregrado, 11 estudiantes de posgrado, 61 docentes universitarios y 27 administrativos académicos; el alfa de Cronbach fue de 0,85 , que indica buena consistencia interna.

\section{Procedimiento}

A continuación, se presenta el procedimiento realizado para evaluar las propiedades psicométricas de la escala EACIN:

1. Administración del instrumento. Una vez obtenidos los permisos de las tres instituciones educativas, se contactó a los participantes y se les leyó el consentimiento informado, en el que se describió el objetivo y la justificación del estudio, la confidencialidad de los datos y la ausencia de riesgos físicos, sociales, laborales o académicos. Igualmente, se informó que al terminar la investigación se les remitiría por correo electrónico el artículo científico, producto de la misma. Una vez firmado el consentimiento los participantes diligenciaron el instrumento individualmente.

En el caso de los estudiantes de bachillerato se remitió previamente el consentimiento a los padres y/o cuidadores. Solo se aplicó a quienes firmaron el asentimiento y sus padres o cuidadores otorgaron el respectivo consentimiento.

2. Análisis estadísticos. Se implementó el análisis paralelo de Horn, que tiene como propósito ajustar el número de factores y de variables por factor sin que se afecte el valor de la prueba. En este análisis se seleccionan los componentes o factores comunes que evidencian valores propios mayores que los que se obtendrían al azar (Brett, Ted, \& Andrys, 2010; Lloret-Segura, Ferretes-Traver, Hernández-Baeza, \& Tomás-Marco, 2014), mediante una sintaxis sencilla propuesta por Thompson y Daniel (1996), que puede ejecutarse con el software estadístico SPSS versión 22,0. Para decidir el número de factores a extraer se identificó el autovalor de los datos reales con magnitud superior al autovalor de los datos simulados. La lógica del procedimiento es que solamente deben interpretarse los factores reales que explican más varianza que los aleatorios (Kahn, 2006). 
Luego se examinó la contribución de cada ítem a la confiabilidad de la prueba, mediante el índice alfa de Cronbach. Asimismo, se examinó la correlación ítem-total corregida, por medio del software estadístico SPSS versión 22,0. Posteriormente, se implementaron las pruebas de adecuación muestral Kaiser-Meyer-Olkin (KMO) y de esfericidad de Bartlett, mediante el software mencionado, para determinar la conveniencia de efectuar un análisis factorial exploratorio de componentes principales, con rotación Promax y normalización Kaiser.

Se determinaron los factores que componen la Escala y los ítems correspondientes, teniendo en cuenta los resultados obtenidos con el análisis paralelo de Horn. Posteriormente, se analizó la consistencia interna de los factores resultantes y de la escala total, con el índice alfa de Cronbach y las correlaciones existentes entre estos factores mediante el índice de correlación de Pearson, con el software estadístico SPSS versión 22,0.

\section{Resultados}

Los resultados del análisis paralelo de Horn sugirieron aceptar solamente tres factores (véase la Tabla 1) y los resultados de la prueba alfa de Cronbach evidenciaron seis ítems (Items: 15, 23, 28, 29, 32 y 33) con una correlación ítem-total corregida inferior a 0,30, que si se eliminaban generaban una confiabilidad del instrumento por encima de 0,854 , por lo que se optó excluirlos de los análisis posteriores.

Tabla 1. Componentes aceptados y rechazados según los resultados del análisis paralelo de Horn

\begin{tabular}{ccccl}
\hline Ítem & Datos real & Media & Percentil 95 & Decisión \\
\hline 1 & 7,57 & 1,57 & 1,64 & Aceptar \\
2 & 4,07 & 1,50 & 1,55 & Aceptar \\
3 & 2,34 & 1,44 & 1,49 & Aceptar \\
4 & 1,37 & 1,40 & 1,43 & Rechazar \\
5 & 1,20 & 1,35 & 1,39 & Rechazar \\
6 & 1,10 & 1,32 & 1,35 & Rechazar \\
7 & 1,06 & 1,28 & 1,31 & Rechazar \\
8 & 1,00 & 1,24 & 1,27 & Rechazar \\
9 & 0,93 & 1,21 & 1,24 & Rechazar \\
10 & 0,86 & 1,18 & 1,21 & Rechazar \\
11 & 0,83 & 1,15 & 1,18 & Rechazar \\
12 & 0,79 & 1,12 & 1,15 & Rechazar \\
13 & 0,74 & 1,09 & 1,12 & Rechazar \\
14 & 0,73 & 1,07 & 1,09 & Rechazar \\
15 & 0,71 & 1,04 & 1,06 & Rechazar \\
16 & 0,70 & 1,01 & 1,04 & Rechazar \\
17 & 0,65 & 0,99 & 1,01 & Rechazar \\
18 & 0,64 & 0,96 & 0,99 & Rechazar \\
19 & 0,59 & 0,94 & 0,96 & Rechazar \\
20 & 0,57 & 0,91 & 0,93 & Rechazar \\
21 & 0,55 & 0,89 & 0,91 & Rechazar \\
22 & 0,51 & 0,87 & 0,89 & Rechazar \\
\hline & & & &
\end{tabular}




\begin{tabular}{ccccl}
\hline Ítem & Datos real & Media & Percentil 95 & Decisión \\
\hline 23 & 0,49 & 0,84 & 0,86 & Rechazar \\
24 & 0,49 & 0,82 & 0,84 & Rechazar \\
25 & 0,46 & 0,79 & 0,82 & Rechazar \\
26 & 0,44 & 0,77 & 0,79 & Rechazar \\
27 & 0,40 & 0,75 & 0,77 & Rechazar \\
28 & 0,39 & 0,72 & 0,75 & Rechazar \\
29 & 0,39 & 0,70 & 0,72 & Rechazar \\
30 & 0,34 & 0,67 & 0,69 & Rechazar \\
31 & 0,33 & 0,65 & 0,67 & Rechazar \\
32 & 0,31 & 0,62 & 0,64 & Rechazar \\
33 & 0,26 & 0,59 & 0,62 & Rechazar \\
34 & 0,20 & 0,55 & 0,58 & Rechazar \\
\hline Nota. Se aceptan los factores cuyo dato real es superior al percentil 95 simulado.
\end{tabular}

Los resultados obtenidos con la medida de adecuación muestral de $\mathrm{KMO}(0,889)$ y la prueba de esfericidad de Bartlett $\left(X^{2}=4371.445, p=0,000\right)$, indicaron la conveniencia de efectuar el análisis factorial, el cual evidenció cinco factores que explicaban el $53.47 \%$ de la varianza. De acuerdo con los resultados del análisis paralelo de Horn, se tuvieron en cuenta los ítems que cargaban en los tres primeros factores.

Con base en el contenido de los ítems agrupados en los tres factores seleccionados, se conformó la Versión Revisada de la EACIN con las siguientes sub-escalas (véase la Tabla 2): (a) desinterés por la investigación (nueve ítems con calificación inversa: 1, 4, 5, 9, 14, 19, 27, 30 y 34); (b) vocación por la investigación (doce ítems con calificación directa: $2,3,6,8,10,11,13,16,17,18,24$ y 25) y (c) valoración de la investigación (siete ítems con calificación directa: 7, 12, 20, 21, 22, 26 y 31). El índice alfa de Cronbach obtenido en cada una de estas sub-escalas fue: (a) desinterés por la investigación: 0,87; (b) vocación por la investigación: 0,87 y (c) valoración de la investigación: 0,77. Para la escala total el alfa fue 0,87. En el Apéndice se presenta el instrumento resultante.

Tabla 2. Análisis factorial con los componentes retenidos mediante el análisis paralelo de Horn.

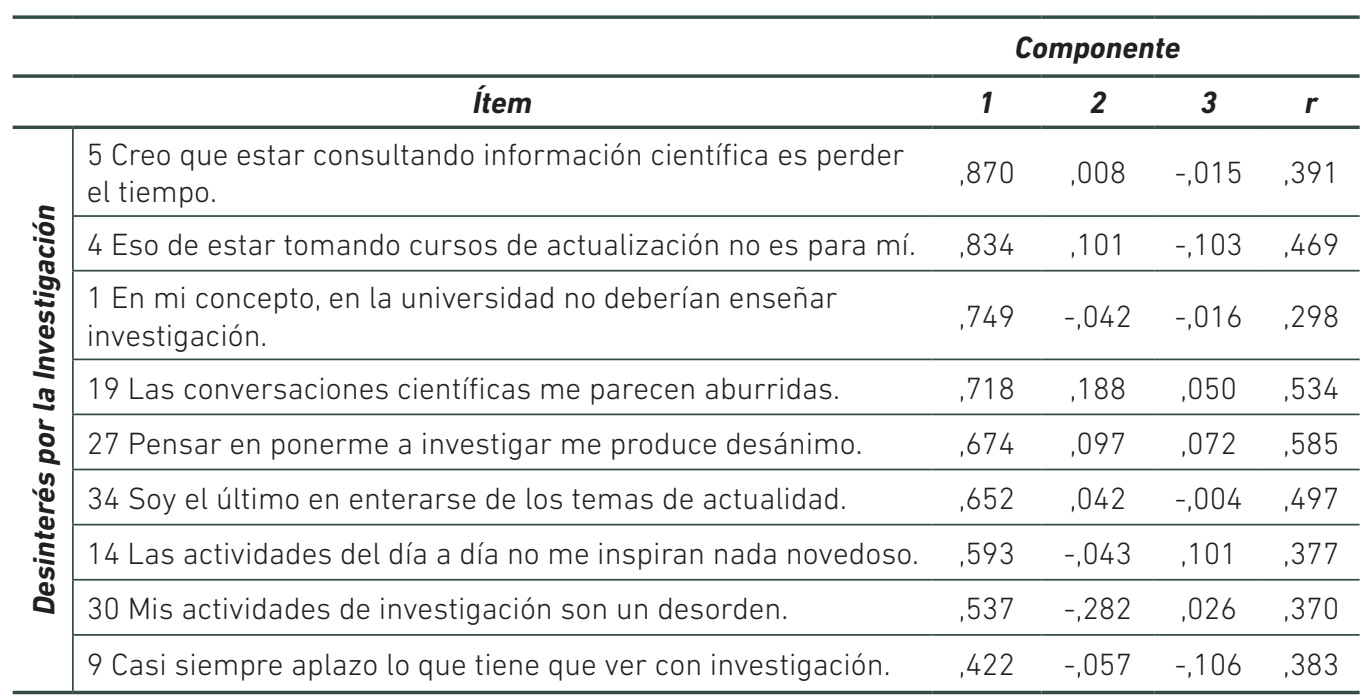




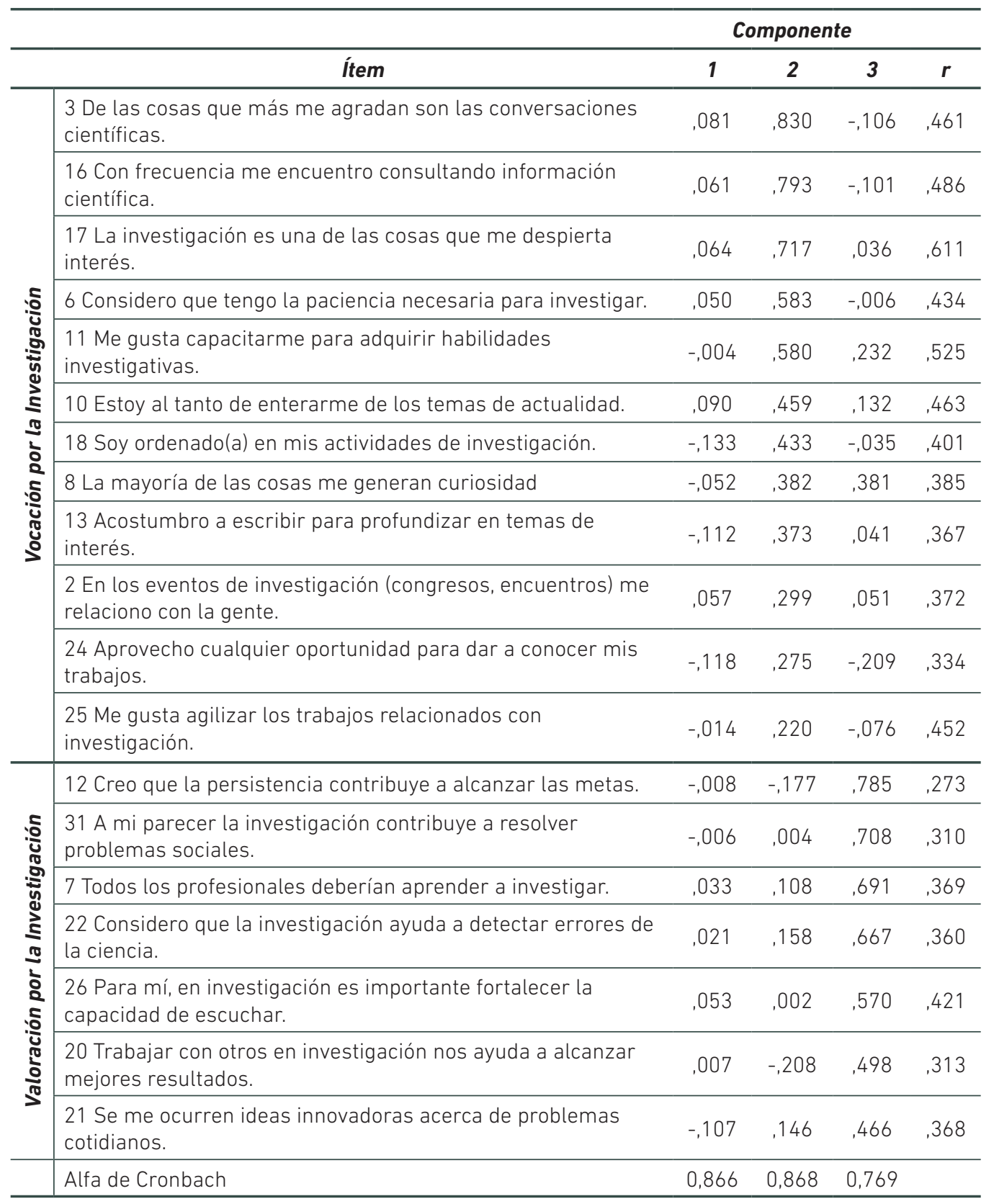

Nota. Método de extracción: Análisis de componentes principales. Método de rotación: Promax con normalización Kaiser. r: Correlación ítem-total corregida.

Las puntuaciones obtenidas en la sub-escala de vocación por la investigación correlacionaron positivamente y de manera estadísticamente significativa con las de la sub-escala de valoración de la investigación $(r=0.510 ; p=0,000)$ y también con la sub-escala de desinterés por la investigación ( $r=0180 ; p=0,000)$. Esta última sub-escala correlacionó con la sub-escala de valoración de la investigación $(r=0,115$; $p=0,018)$.

\section{Discusión}

Este estudio tuvo como objetivo examinar las propiedades psicométricas de la EACIN en una muestra colombiana. Los resultados obtenidos con el análisis paralelo de Horn y el análisis factorial sugirieron la conformación de una versión revisada del instrumento, constituida por tres factores cuyos ítems se agruparon en tres sub-escalas: desinterés 
Pág 98

La versión revisada y cada uno de sus componentes o factores evidenciaron un buen nivel de consistencia interna; correlacionaron positivamente y de manera estadísticamente significativa las subescalas de vocación por la investigación y valoración de la investigación, con una fuerza de correlación alta, que indica que miden aspectos relacionados, mientras que estas evidenciaron una ausencia de correlación con la sub-escala de desinterés por la investigación, que sugiere que miden aspectos diferentes (interés versus desinterés por la investigación). por la investigación, vocación por la investigación y valoración de la investigación. La versión revisada y cada uno de sus componentes o factores evidenciaron un buen nivel de consistencia interna; correlacionaron positivamente y de manera estadísticamente significativa las subescalas de vocación por la investigación y valoración de la investigación, con una fuerza de correlación alta, que indica que miden aspectos relacionados, mientras que estas evidenciaron una ausencia de correlación con la sub-escala de desinterés por la investigación, que sugiere que miden aspectos diferentes (interés versus desinterés por la investigación).

La versión revisada de la Escala EACIN obtuvo un valor alfa de 0,87 que indica una buena consistencia interna, además presenta un valor más alto que la versión de Aldana et al. $\underline{\text { (2016) }}$ que fue de 0,85. Este resultado es coherente con lo hallado en México por Quezada-Berumen, Moral de la Rubia y Landero-Hernández (2019), en un estudio en el que se validó la misma escala en estudiantes de Psicología de una universidad privada mexicana y se identificaron tres factores: aspectos afectivos, y conductuales y cognoscitivos, ambos con direccionalidad positiva y consistencia interna de 0,90 y 0,89, respectivamente, y un tercer factor denominado aspectos conductuales y afectivos, con direccionalidad inversa y consistencia de 0,87.

Las sub-escalas vocación por la investigación y valoración de la investigación de la EACIN-R son coherentes con las de otros instrumentos que incluyen medidas similares, como la Escala de Actitudes hacia la Investigación (Attitudes Toward Research Scale)) de Papanastasiou (2005), que contiene una escala de valor del conocimiento científico. Así mismo, estas sub-escalas son similares a las reportadas por Castro (2017), que miden actitud hacia la formación investigativa e incorpora una escala de satisfacción-agrado por la investigación.

Sin embargo, ninguno de los instrumentos revisados contiene un componente o sub-escala relacionada con el desinterés por la investigación, que puede utilizarse con diferentes propósitos, como clasificar estudiantes o docentes con miras a vincularlos a semilleros y/o proyectos de investigación, así como para evaluar los resultados de procesos de formación investigativa.

Respecto a la validez del instrumento, se recomienda explorar la validez de criterio concurrente mediante pruebas validadas que midan actitudes hacia la investigación. Igualmente, es necesario hallar la validez de criterio tipo predictivo, con el fin de examinar la capacidad del instrumento para predecir la predisposición hacia asignaturas de investigación y demás actividades relacionadas con el tema.

Asimismo, es necesario realizar un análisis factorial de la estructura evidenciada mediante el análisis factorial exploratorio y examinar la validez convergente y discriminante del instrumento. Una vez se cuente con índices adecuados de confiabilidad y validez se sugiere obtener baremos, teniendo en cuenta variables como la edad, sexo, nivel educativo y rol.

Entre las fortalezas del presente estudio cabe mencionar lo siguiente: la participación de hombres y mujeres, estudiantes de 13 carreras profesionales, docentes, administrativos académicos y estudiantes de último grado de bachillerato; un tamaño muestral significativamente más amplio que el utilizado en la versión anterior: 427 y 187 participantes, respectivamente. 
La aplicación del análisis paralelo de Horn, considerado como una de las mejores alternativas para la extracción de número de factores, que arrojó como resultado un instrumento más corto, confiable y de más fácil aplicación, con los ítems agrupados en tres sub-escalas, que responden a nominaciones conceptuales relacionadas con su contenido; más allá de la estructura del modelo multidimensional de la versión anterior, que divide el comportamiento en tres factores: afectivo, cognitivo y conductual. Cada una de las tres sub-escalas contiene un número de ítems suficiente para medir el componente. El resultado del índice alfa de Cronbach de las sub-escalas es coherente con el alfa total $(0,87)$, que subió con respecto al valor del alfa de la versión original de la $\operatorname{EACIN}(0,85)$.

Las limitaciones se relacionan con la falta de participación de estudiantes de posgrado, que puede limitar la generalización de los resultados a estos actores y el hecho de que la selección de los participantes no fue aleatoria, que podría dar lugar a sesgos referentes a la autoselección, generados por las motivaciones para participar en la investigación, aspectos que deberían considerarse en los próximos estudios del instrumento.

\section{Agradecimientos}

Se agradece al profesor Edgar Antonio Ibañez Pinilla el apoyo estadístico.

\section{Referencias}

Akçöltekin, A. (2016). Investigation of the effect of training on the development of high school teachers' attitudes towards scientific research and project competitions. Educational Sciences: Theory \& Practice, 16(4) - 1349-1380. Recuperado de: https://eric.ed.gov/?id=EJ1115068

Aldana, G. M., \& Joya, N. S. (2011). Actitudes hacia la investigación científica en docentes de metodología de la investigación. Tabula Rasa, 14, 295-309. Recuperado de: https://www.revistatabularasa.org/numero14/actitudes-hacia-la-investigacion-cientifica-en-docentes-de-metodologia-de-la-investigacion/

Aldana, G. M., Caraballo, G. J., \& Babativa, D. A. (2016). Escala EACIN para medir actitudes hacia la investigación: validación de contenido y confiabilidad. Aletheia, 8(2), 104-126. Recuperado de: https://aletheia.cinde.org.co/index.php/ALETHEIA/article/view/325

Alonso, J., Alonso, A., \& Valadez, D. (2015). Actitud hacia la investigación científica en estudiantes de Enfermería. El Arte del Cuidado, 4(7), 21-35. Recuperado de: http://www.revistas.unam.mx/index.php/cuidarte/article/view/69106/60936

Allport, G. W. (1935). Attitudes. En C. Murchiso (Ed.), A handbook of social psychology (pp. 798-844). Worcester, MA, US: Clark University Press.

Arellano Torres, A., Gaeta González, M. L.; Peralta López, F., \& Cavazos Arroyo, J. (2019). Actitudes hacia la discapacidad en una universidad mexicana. Revista Brasileira de Educação, 24, 1- 20. doi: http://dx.doi.org/10.1590/s1413-24782019240023

Arias, F. (2012). Introducción a la metodología científica (6 ${ }^{a}$ Ed.). Caracas: Espíteme.

Blanco, N., \& Alvarado, M. E. (2005). Escala de actitud hacia el proceso de investigación científico social. Revista de Ciencias Sociales, 11(3), 537-544. Recuperado de: https://www.redalyc.org/articulo.oa?id=28011311

Brett, W., Brown, T., \& Onsman, A. (2010). Exploratory factor analysis: A five-step guide for novices. Australasian Journal of Paramedicine, 8(3). Recuperado de: http:// ro.ecu.edu.au/jephc/vol8/iss3/1

Castro, S. P. (2017). Diseño y validación de un instrumento para evaluar la actitud hacia la investigación formativa en estudiantes universitarios. Actualidades Pedagógicas, 70, 165-182. doi: https://dx.doi.org/10.19052/ap.3996 
De la Cruz, C. (2013). Actitudes hacia la investigación científica en estudiantes universitarios: Análisis en dos universidades nacionales de Lima. Psiquemag, 2(1), 1-16. Recuperado de: http://ojs.ucvlima.edu.pe/index.php/psiquemag/article/ view/12/12

De las Salas, M., Perozo, S., \& Lago, Z. (2014). Actitud del estudiante universitario hacia la investigación en el núcleo Luz - Costa Oriente del Lago. Revista Electrónica de Humanidades, Educación y Comunicación Social, 18, 162 - 176. Recuperado de: http://ojs.urbe.edu/index.php/redhecs/article/view/2446/2258

Díaz, C., Manrique, L. M., Galán, E., \& Apolaya, M. (2008). Conocimientos, actitudes y prácticas en estudiantes pregrado de facultades de medicina del Perú. Acta Médica Peruana, 25(1), 9-15. Recuperado de: http://www.scielo.org.pe/pdf/amp/ v25n1/a03v25n1.pdf

Hacieminoglu, E. (2016). Elementary school students' attitude toward science and related variables. International Journal of Environmental \& Science educatión, 11 (2), 35-52. doi: https://doi.org/10.12973/ijese.2016.288a

Kahn, J. H. (2006). Factor analysis in counseling psychology research, training and practice. The Counseling Psychologist, 34, 1-36. doi: https://doi. org/10.1177/0011000006286347

Kennedy, J. P., Quinn, F., \& Taylor, N. (2016). The school science attitude survey: A new instrument for measuring attitudes towards school science. International Journal of Research \& Method in Education, 39(4), 422 - 445. doi: https://doi.org/10.1 $\underline{080 / 1743727 X .2016 .1160046}$

Korkmaz, O., Sahin, A., \& Yeșil, R. (2011). Study of validity and reliability of Scale of Attitude towards Scientific Research. Elementary Education Online, 10(3), 961-973. Recuperado de: http://ilkogretim-online.org.tr/index.php/io/article/ view/1569/1405

Lloret-Segura, S., Ferretes-Traver, A., Hernández-Baeza, A., \& Tomás-Marco, I. (2014). Análisis factorial exploratorio de los ítems: una guía práctica, revisada y actualizada. Anales de psicología, 30(3), 1151 - 1169. doi: http://dx.doi.org/10.6018/ analesps.30.3.199361

Mamani, 0.J. (2011). Actitud hacia la investigación y su importancia en la elección de la modalidad de tesis para optar el título profesional. Revista Científica de Ciencias de la Salud, 4(4), 22 - 27. doi: https://doi.org/10.17162/rccs.v4i1.82

May, M. H., \& Hong, Z. (2015). Unpacking the paradox of Chinese science learners: insights from research into Asian Chinese school students' attitudes towards learning science, science learning strategies, and scientific epistemological views. Studies in Science Education, 52, 29-62. doi: https://doi.org/10.1080/0305 7267.2015 .1112471

Montero, I., \& León, O. (2007). A guide for naming research studies in psychology. International Journal of Clinical and Health Psychology, 7(3), 847-862. Recuperado de: https: / / www.redalyc.org/articulo.oa?id=33770318

Nobigrot-Kleinman, D., Nobigrot-Streimbleinsky, M., \& Galván-Huerta, S. C. (1995). Las actitudes hacia la investigación y el aprendizaje en estudiantes de medicina, 1984-1994. Salud Pública de México, 37(4), 316-322. Recuperado de: http://saludpublica.mx/index.php/spm/article/view/5851

Norman, G. R., \& Streiner, D. L. (1996). Bioestadística. Madrid: Harcourt.

Ortega Carrasco, R. J., Veloso Toledo, R. D., \& Hansen, O. S. (2018). Percepción y actitudes hacia la investigación científica. Revista de Investigación en Ciencias Sociales y Humanidades, 5(2), 101 - 108. doi: https://dx.doi.org/10.30545/academo.2018.jul-dic.2

Osman, T. (2016). Medical students' perceptions towards research at a Sudanese University. BMC Medical Education, 16, 253. doi: https://doi.org/10.1186/s12909016-0776-0 
Papanastasiou, E. C., \& Schumacker, R. (2014). Rasch rating scale analysis of the Attitudes Toward Research Scale. Journal of Applied Measurement, 15(2), 189-99. Recuperado de: https://www.ncbi.nlm.nih.gov/pubmed/24950536

Papanastasiou, E. C. (2005). Factor structure of the "Attitudes Toward Research" Scale. Statistics Education Research Journal, 4(1), 16-26. Recuperado de: https:// iase-web.org/documents/SERJ/SERJ4(1) Papanastasiou.pdf

Quezada-Berumen, L., Moral de la Rubia, J., \& Landero-Hernández, R. (2019). Validación de la Escala de Actitud hacia la Investigación en estudiantes mexicanos de psicología. Revista Evaluar, 19(1), 1-16. Recuperado de: https://revistas.unc. edu.ar/index.php/revaluar/article/view/23874

Rodríguez, O., \& Mora, S. E. (2016). Análisis psicométrico del instrumento actitudes hacia las matemáticas mediante el modelo de Respuesta Graduada de Samejima. Actualidades en Psicología, 30(120), 7-30. doi: http://dx.doi.org/10.15517/ ap.v30i120.18722

Rojas, H. M., Méndez, R., \& Rodríguez, A. (2012). Índice de actitud hacia la investigación en estudiantes del nivel de pregrado. Entramado, 8(2), 216-229. Recuperado de: https://dialnet.unirioja.es/servlet/articulo?codigo $=4265852$

Thompson, B., \& Daniel, L. G. (1996). Factor analytic evidence for the construct validity of scores: An historical overview and some guidelines. Educational and Psychological Measurement, 56, 197-208. doi: https://doi.org/10.1177/0013164496056002001

Van Aalderen-Smeets, S., Walma van der Molen, J. H., van Hest, E. G. C. M., \& Poortman, C. L. (2017). Primary teachers conducting inquiry projects: Effects on attitudes towards teaching science and conducting inquiry. International Journal of Science Education, 39(2), 238-256. doi: https://doi.org/10.1080/09500693.2016.1277280

Veliz, C. D. (2017). Las actitudes hacia la investigación científica y la disposición para la realización de la tesis en estudiantes de Posgrado de dos Universidades de Lima 2017. Tesis doctorado en Ciencias Educación. Recuperado de: http://repositorio. une.edu.pe/bitstream/handle/UNE/2719/TD\%20CE\%202024\%20V1\%20-\%20 Veliz\%20Manrique\%20Cesar\%20David.pdf? sequence=1\&isAllowed=y

Verkade, H., \& Hoon, S. (2016). Undergraduate science students' attitudes toward and approaches to scientific reading and 2016 writing. Research and Teaching, 45(4), 84-89. Recuperado de: https://eric.ed.gov/?id=EJ1095176 


\section{Apéndice}

Escala de actitudes hacia la investigación - versión revisada (EACIN-R)

\begin{tabular}{|c|c|c|c|c|c|c|c|}
\hline \multicolumn{8}{|c|}{$\begin{array}{l}\text { A continuación, encontrará una serie de afirmaciones relacionadas con la investigación, por favor } \\
\text { marque con una X la respuesta con la cual se sienta más identificado(a). No medite mucho su } \\
\text { respuesta, no hay respuestas buenas ni malas. Las opciones son: }\end{array}$} \\
\hline \multicolumn{2}{|c|}{$\begin{array}{l}0 \text { Muy en desacuerdo } \\
1 \text { En desacuerdo } \\
2 \text { Ni de acuerdo ni en desacuerdo }\end{array}$} & \multicolumn{6}{|l|}{$\begin{array}{l}3 \text { De acuerdo } \\
4 \text { Muy de acuerdo }\end{array}$} \\
\hline $\mathrm{N}^{0}$ & \multicolumn{2}{|c|}{ ÍTEMS } & 0 & 1 & 2 & 3 & 4 \\
\hline 1 & \multicolumn{2}{|c|}{$\begin{array}{l}\text { En los eventos de investigación (congresos, encuentros) me relaciono } \\
\text { con la gente. }\end{array}$} & & & & & \\
\hline 2 & \multicolumn{2}{|c|}{ En mi concepto en la universidad no deberían enseñar investigación. } & & & & & \\
\hline 3 & \multicolumn{2}{|c|}{ De las cosas que más me agradan son las conversaciones científicas. } & & & & & \\
\hline 4 & \multicolumn{2}{|c|}{ Eso de estar tomando cursos de actualización no es para mí. } & & & & & \\
\hline 5 & \multicolumn{2}{|c|}{ Creo que estar consultando información científica es perder el tiempo. } & & & & & \\
\hline 6 & \multicolumn{2}{|c|}{ Considero que tengo la paciencia necesaria para investigar. } & & & & & \\
\hline 7 & \multicolumn{2}{|c|}{ Todos los profesionales deberían aprender a investigar. } & & & & & \\
\hline 8 & \multicolumn{2}{|c|}{ La mayoría de las cosas me generan curiosidad. } & & & & & \\
\hline 9 & \multicolumn{2}{|c|}{ Casi siempre aplazo lo que tiene que ver con investigación. } & & & & & \\
\hline 10 & \multicolumn{2}{|c|}{ Estoy al tanto de enterarme de los temas de actualidad. } & & & & & \\
\hline 11 & \multicolumn{2}{|c|}{ Me gusta capacitarme para adquirir habilidades investigativas. } & & & & & \\
\hline 12 & \multicolumn{2}{|c|}{ Creo que la persistencia contribuye a alcanzar las metas. } & & & & & \\
\hline 13 & \multicolumn{2}{|c|}{ Acostumbro a escribir para profundizar en temas de interés. } & & & & & \\
\hline 14 & \multicolumn{2}{|c|}{ Las actividades del día no me inspiran nada novedoso. } & & & & & \\
\hline 15 & \multicolumn{2}{|c|}{ Con frecuencia me encuentro consultando información científica. } & & & & & \\
\hline 16 & \multicolumn{2}{|c|}{ La investigación es una de las cosas que me despierta interés. } & & & & & \\
\hline 17 & \multicolumn{2}{|c|}{ Soy ordenado(a) en mis actividades de investigación. } & & & & & \\
\hline 18 & \multicolumn{2}{|c|}{ Las conversaciones científicas me parecen aburridas. } & & & & & \\
\hline 19 & \multicolumn{2}{|c|}{$\begin{array}{l}\text { Trabajar con otros en investigación nos ayuda a alcanzar mejores } \\
\text { resultados. }\end{array}$} & & & & & \\
\hline 20 & \multicolumn{2}{|c|}{ Se me ocurren ideas innovadoras acerca de problemas cotidianos. } & & & & & \\
\hline 21 & \multicolumn{2}{|c|}{ Considero que la investigación ayuda a detectar errores de la ciencia. } & & & & & \\
\hline 22 & \multicolumn{2}{|c|}{ Aprovecho cualquier oportunidad para dar a conocer mis trabajos. } & & & & & \\
\hline 23 & \multicolumn{2}{|c|}{ Me gusta agilizar los trabajos relacionados con investigación. } & & & & & \\
\hline 24 & \multicolumn{2}{|c|}{$\begin{array}{l}\text { Para mí, en investigación es importante fortalecer la capacidad de } \\
\text { escuchar. }\end{array}$} & & & & & \\
\hline 25 & \multicolumn{2}{|c|}{ Pensar en ponerme a investigar me produce desánimo. } & & & & & \\
\hline 26 & \multicolumn{2}{|c|}{ Mis actividades de investigación son un desorden. } & & & & & \\
\hline 27 & $\begin{array}{l}\text { A mi parecer la investigación } \\
\text { sociales. }\end{array}$ & ye a resolver problemas & & & & & \\
\hline 28 & Soy el último en enterarse de & s de actualidad & & & & & \\
\hline
\end{tabular}




\section{Características de la Escala de Actitudes hacia la Investigación - Versión Revi- sada (EACIN-R)}

La Escala de Actitudes hacia la Investigación - Versión Revisada (EACIN-R) quedó conformada por 28 ítems. Nueve corresponden a la sub-escala Desinterés por la investigación con calificación inversa $(2,4,5,9,14,18,25,26$ y 28), doce a Vocación por la investigación calificación directa $(1,3,6,8,10,11,13,15,16,17,22,23)$ y siete a Valoración de la investigación calificación directa $(7,12,19,20,21,24,27)$. El intervalo de puntuación total es de 0 a 112. Puntajes altos indican actitud positiva hacia la investigación y puntajes bajos indican una actitud desfavorable hacia la misma. Las opciones de respuesta se valoran de la siguiente forma:

$0=$ Muy en desacuerdo

$1=$ En desacuerdo

$2=$ Ni de acuerdo ni en desacuerdo

$3=$ De acuerdo

4= Muy de acuerdo 